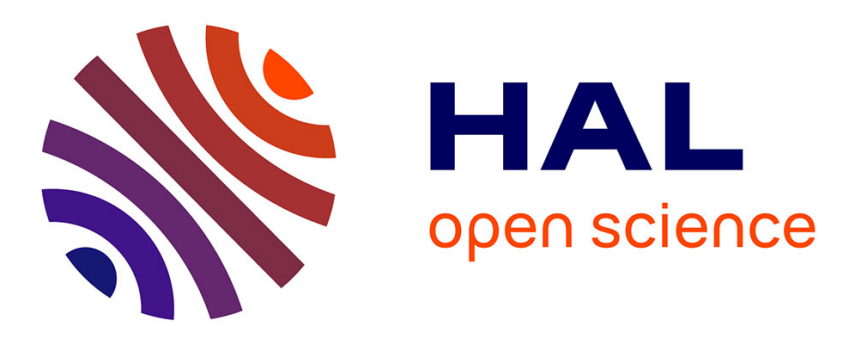

\title{
Modélisation des régimes transitoires des machines asynchrones sans fer à induit massif
}

A. Diop, G. Quichaud

\section{To cite this version:}

A. Diop, G. Quichaud. Modélisation des régimes transitoires des machines asynchrones sans fer à induit massif. Revue de Physique Appliquée, 1988, 23 (1), pp.79-89. 10.1051/rphysap:0198800230107900 . jpa-00245750

\section{HAL Id: jpa-00245750 https://hal.science/jpa-00245750}

Submitted on 1 Jan 1988

HAL is a multi-disciplinary open access archive for the deposit and dissemination of scientific research documents, whether they are published or not. The documents may come from teaching and research institutions in France or abroad, or from public or private research centers.
L'archive ouverte pluridisciplinaire HAL, est destinée au dépôt et à la diffusion de documents scientifiques de niveau recherche, publiés ou non, émanant des établissements d'enseignement et de recherche français ou étrangers, des laboratoires publics ou privés. 


\title{
Modélisation des régimes transitoires des machines asynchrones sans fer à induit massif
}

\author{
A. Diop (*) et G. Quichaud \\ Laboratoire d'Electrotechnique des Universités Paris VI et XI, U.A. CNRS 845, Bât. 214, 91405 Orsay, \\ France
}

(Reçu le 18 avril 1987, révisé le 29 juillet et le 24 septembre 1987, accepté le 2 octobre 1987)

\begin{abstract}
Résumé. - Les auteurs présentent une modélisation numérique des courants induits dans un conducteur massif amagnétique en rotation ou en translation dans un champ magnétique d'excitation. Les courants induits sont considérés comme une superposition d'ondes propres. L'intérêt réside dans le fait qu'à chacune de ces ondes, caractérisée par une répartition spatiale et une constante de temps on peut associer le circuit équivalent d'une machine asynchrone idéale. On donne enfin des applications de cette méthode à des génératrices impulsionnelles et à un lanceur électromagnétique.
\end{abstract}

\begin{abstract}
The authors present a numerical modelling of the induced currents in a non magnetic solid conductor in rotation or translation in an applied magnetic field. The induced currents are regarded as superposing of proper waves. The interest is all in the fact that to each of this waves characterized by a space distribution, a time constant, we can associate the equivalent circuit of an ideal asynchronous machine. Finally we apply this method to pulsed generators and electromagnetic launcher.
\end{abstract}

\section{Introduction.}

Dans le cadre de l'électrotechnique impulsionnelle un certain nombre de dispositifs asynchrones sans fer (génératrices impulsionnelles, lanceurs électromagnétiques) sont constitués d'une excitation polyphasée et d'un induit amagnétique massif de révolution $[1,12]$.

La figure 1 donne la structure de principe d'une génératrice impulsionnelle [1]. L'induit massif $D$ est en rotation à la vitesse angulaire $\Omega$ autour de son axe $\mathrm{O} z$ de révolution. Cet induit est placé à l'intérieur d'un inducteur $D_{0}$ polyphasé à $2 \mathrm{p}$ poles. On notera $\mathbf{i}_{0}$ la répartition de densité de courant inducteur dans $\mathrm{D}_{0}$. Cette génératrice est de type cylindrique [2] lorsque le rotor étant relativement long, l'inducteur (en pointillé) crée un champ magnétique utile radial. Elle est de type discoïde [7] lorsque le rotor étant mince, l'inducteur (en trait plein) crée un champ à prépondérance axiale. Dans les deux cas le rotor possède un plan médian $\Delta$ de symétrie perpendiculaire à son axe de rotation. Ce plan médian est un plan de symétrie pour les courants d'excitation dans

(*) Actuellement service IMA DER EDF Clamart, France.

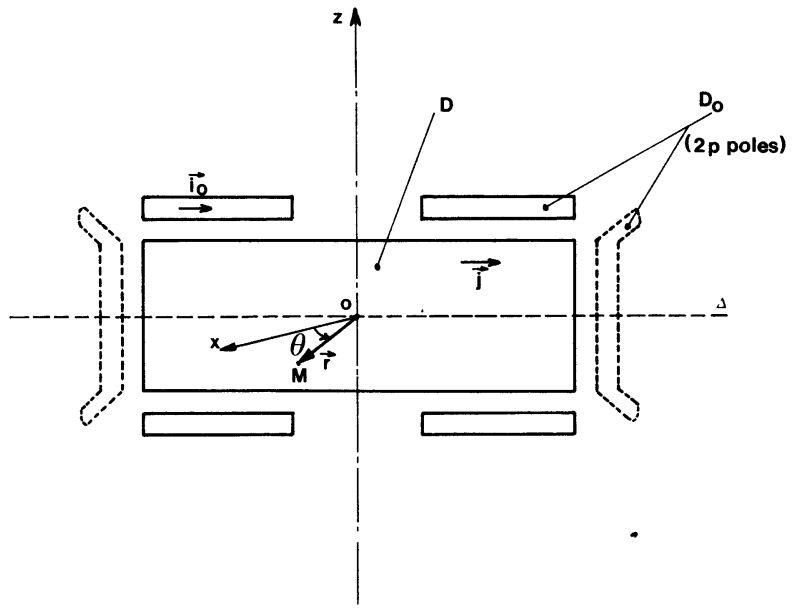

Fig. 1. - Génératrices impulsionnelles.

[Pulsed generators.]

le cas d'une machine discoïde et un plan d'antisymétrie dans le cas cylindrique.

La figure 2 donne le schéma de principe d'un lanceur électromagnétique de type asynchrone [12]. L'induit $\mathrm{D}$, placé à l'intérieur de bobines inductrices circulaires contenues dans $\mathrm{D}_{0}$, est en translation à la 


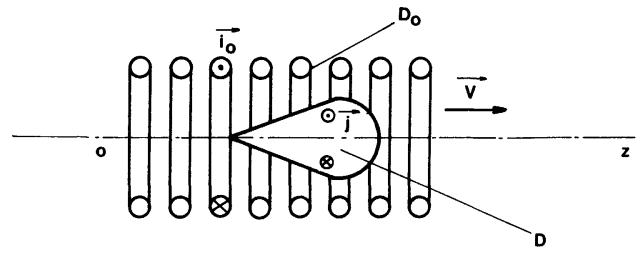

Fig. 2. - Lanceur électromagnétique.

[Electromagnetic launcher.]

vitesse $V$ parallèlement à son axe de révolution. Un cas idéal [19] de lanceur est celui où l'induit est un cylindre infiniment long de rayon $R$, les bobines excitatrices parcourues par des courants polyphasés ayant une répartition périodique en $z$ de période $\frac{2 \pi}{k}$.

Les fonctionnements impulsionnels de ces dispositifs $[12,3]$ conduisent à l'étude de régimes transitoires électromécaniques. Nous allons montrer dans la suite l'intérêt qu'il peut y avoir pour cette étude à considérer les courants induits comme superposition de modes propres de diffusion dans le volume conducteur $D$.

Notons qu'on est également amené à modéliser de tels courants induits dans les écrans des alternateurs à inducteurs supraconducteurs lors de leurs régimes transitoires [9].

2. Modes propres de diffusion des courants $[15-17$, 9].

Nous appellerons répartition de densité de courant admissible dans un induit massif amagnétique $D$ toute répartition $\mathbf{j}$ de densité de courant vérifiant $\operatorname{div} \mathbf{j}=\mathbf{0}$ dans $\mathrm{D}$ et dont la composante normale $\mathbf{j} \cdot \mathbf{n}$ est nulle sur la frontière de $\mathrm{D}$. Précisons [8] que cet ensemble $\mathcal{H}$ de répartitions définies dans $\mathrm{D}$, muni du produit scalaire fonctionnel :

$$
\left\langle\mathbf{j}_{1}, \mathbf{j}_{2}\right\rangle=\int_{\mathrm{D}} \mathbf{j}_{1} \cdot \mathbf{j}_{2} \mathbf{d} \tau
$$

est un espace de Hilbert. Lorsque l'induit sera le domaine infini de la figure 3 nous ne considérerons que les répartitions de période $\frac{2 \pi}{k}$ en $z$ et les volumes $D$ et $D_{0}$ seront limités dans la direction $\mathrm{O} z$ à une telle période.

Nous utiliserons dans D comme détermination du potentiel vecteur magnétique d'une répartition de courant $\mathbf{j}^{\prime}$ définie dans $\mathrm{D}^{\prime}$, la quantité $\overline{\bar{a}}_{\mathrm{D}^{\prime}}\left(\mathbf{j}^{\prime}\right)$ ainsi définie :

$$
\begin{aligned}
& \overline{\bar{a}}_{\mathrm{D}^{\prime}}\left(\mathbf{j}^{\prime}\right)=\mathbf{a}^{\prime}+\operatorname{grad} \varphi^{\prime} \mid \\
& \Delta \varphi^{\prime}=0 \\
& \frac{\partial \varphi^{\prime}}{\partial n}+\mathbf{a}^{\prime} \cdot \mathbf{n}=0 \quad \text { dans } \mathrm{D} \\
& \text { sur la frontière de D }
\end{aligned}
$$

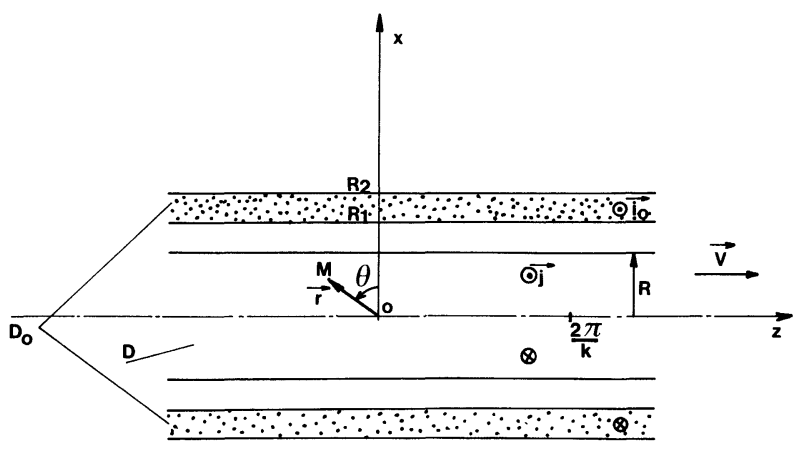

Fig. 3. - Lanceur électromagnétique idéal.

[Ideal electromagnetic launcher.]

où $\mathbf{a}^{\prime}$ est le potentiel vecteur magnétique classique de la magnétostatique :

$$
\mathbf{a}^{\prime}=\frac{\mu_{0}}{4 \pi} \int_{\mathbf{D}^{\prime}} \frac{\mathbf{j}^{\prime} \mathrm{d} \tau}{r} .
$$

Remarquons qu'à un facteur dimensionnel près cette détermination du potentiel vecteur est un élément de $\mathcal{H}$. Le produit scalaire (1) permet d'interpréter $\overline{\bar{a}}_{\mathrm{D}^{\prime}}\left(\mathbf{j}^{\prime}\right)$ comme une projection orthogonale de $\mathbf{a}^{\prime}$ dans $H$. Cela résulte de la nullité de l'intégrale $\int_{D} \operatorname{grad} \varphi^{\prime} \cdot \mathbf{j} \mathrm{d} \tau$ quelle que soit la répartition $\mathbf{j}$ appartenant à $\mathcal{H}$. Dans la suite nous simplifierons les notations en écrivant $\overline{\bar{a}}(\mathbf{j})$ et $\overline{\bar{a}}_{0}\left(\mathbf{i}_{0}\right)$ à la place de $\overline{\bar{a}}_{\mathrm{D}}(\mathbf{j})$ et $\overline{\bar{a}}_{\mathrm{D}_{0}}\left(\mathbf{i}_{0}\right)$.

Avec ces définitions et si on se place dans un référentiel lié à $D$ la répartition $\mathbf{j}$ de densité de courant induit dans D par $\mathbf{i}_{0}$ est la solution dans $\mathcal{H}$ de l'équation fonctionnelle suivante :

$$
\mathbf{j}=-\gamma \frac{\partial \overline{\bar{a}}(\mathbf{j})}{\partial t}-\gamma \frac{\partial \overline{\bar{a}}_{0}\left(\mathbf{i}_{0}\right)}{\partial t}
$$

associée à des conditions initiales. $\gamma$ est la conductivité de l'induit.

On appelle mode propre de diffusion du courant dans D une solution de (4) due aux seules conditions initiales $\left(i_{0}=0\right)$ et se mettant sous la forme :

$$
\mathbf{j}=\mathbf{j}_{n} \exp -\frac{t}{\tau_{n}}
$$

La répartition $\mathbf{j}_{n}$ indépendante du temps est donc solution de l'équation homogène :

$$
\mathbf{j}_{n}=\frac{\gamma}{\tau_{n}} \overline{\bar{a}}\left(\mathbf{j}_{n}\right) .
$$

Ainsi $\lambda_{n}=\frac{\tau_{n}}{\gamma}$ et $\mathbf{j}_{n}$ sont les valeurs propres et les fonctions propres de l'opérateur linéaire $\overline{\bar{a}}$. On montre [8] que cet opérateur est auto-adjoint, défini positif et compact. Dans ces conditions ses valeurs 
propres sont réelles positives bornées dénombrables avec zéro comme point d'accumulation et peuvent donc être ainsi classées :

$$
\lambda_{1} \geqslant \lambda_{2} \geqslant \lambda_{3} \cdots \geqslant \lambda_{n} \geqslant \cdots>0 .
$$

Les répartitions $\mathbf{j}_{n}$ indépendantes $\mathrm{du}$ temps où modes propres de diffusion associées à ces valeurs propres $\lambda_{n}$ et telles que :

$$
\overline{\bar{a}}\left(\mathbf{j}_{n}\right)=\lambda_{n} \mathbf{j}_{n}
$$

sont orthogonales et forment une base de $\mathcal{H}$ que nous supposerons orthonormée dans la suite.

\section{Décomposition d'une répartition induite en modes propres.}

Les $\mathbf{j}_{n}$ formant une base de $\mathscr{H}$ on peut rechercher la solution complète de (4) sous la forme de la série :

$$
\mathbf{j}=\sum_{n=1}^{\infty} c_{n}(t) \mathbf{j}_{n}
$$

Ainsi en portant cette série dans (4) on est conduit à déterminer chaque coefficient $c_{n}(t)$ comme solution de l'équation différentielle :

$$
\tau_{n} \frac{\mathrm{d} c_{n}}{\mathrm{~d} t}+c_{n}=-\gamma \frac{\mathrm{d} a_{0 n}}{\mathrm{~d} t}
$$

où

$$
a_{0 n}=\int_{\mathrm{D}} \overline{\bar{a}}_{0}\left(\mathbf{i}_{0}\right) \cdot \mathbf{j}_{n} \mathrm{~d} \tau=\int_{\mathrm{D}} \mathbf{a}_{0} \cdot \mathbf{j}_{n} \mathrm{~d} \tau
$$

$\mathbf{a}_{0}$ est le potentiel vecteur classique de la magnétostatique donné par (3) et dû à la répartition $\mathbf{i}_{0}$ de $\mathrm{D}_{0}$.

Les $\mathbf{j}_{n}$ formant une base orthonormée de J la puissance dissipée par effet joule dans l'induit se simplifie sous la forme :

$$
P_{\mathrm{J}}(t)=\sum_{n=1}^{\infty} \frac{1}{\gamma} c_{n}^{2}(t) .
$$

Des considérations de symétrie de l'induit et de l'inducteur conduisent au fait que seuls certains modes sont excités $\left(a_{0 n} \neq 0\right)$. Ainsi dans le cas des lanceurs des figures 2 et 3 les modes excités ont, en coordonnées cylindriques, une seule composante $j_{\theta}$ non nulle.

\section{Ondes propres harmoniques de polarité $p$.}

Nous allons considérer dans la suite le cas des dispositifs possédant un induit invariant par rotation (Fig. 1) ou translation (Fig. 3) et une densité de courant d'excitation $i_{0}$ périodique dans le sens de ce déplacement. Pour de tels dispositifs nous allons montrer qu'il est intéressant de grouper dans la série
(9) les contributions des modes ayant la même constante de temps. Ce groupement ayant une interprétation simple vu des bornes d'alimentation de l'inducteur nous le nommerons onde propre harmonique. Nous allons d'abord nous limiter au cas des génératrices de la figure 1 puis transposerons les résultats au cas du lanceur idéal de la figure 3 . Nous nous plaçons en coordonnées cylindriques $(r, \theta, z)$.

L'invariance du rotor lors d'une rotation conduit au fait que chaque valeur propre $\lambda_{n}$ est double. Les deux modes propres indépendants associés ont la forme suivante :

$$
\begin{aligned}
& \mathbf{j}_{n}^{\prime}=\mathbf{J}_{n}(r, z) \cos \ell_{p} \boldsymbol{\theta} \\
& \mathbf{j}_{n}^{\prime \prime}=\mathbf{J}_{n}(r, z) \sin \ell_{p} \boldsymbol{\theta}
\end{aligned}
$$

où $\ell$ est un entier positif et

$$
\int_{d} J_{n}^{2} r \mathrm{~d} r \mathrm{~d} z=\frac{1}{\pi}
$$

car $\mathbf{j}_{n}^{\prime}$ et $\mathbf{j}_{n}^{\prime \prime}$ sont des éléments de $\mathscr{H}$ dont la norme est égale à l'unité. $d$ est la section méridienne de $\mathrm{D}$. Ces modes n'étant excités que par l'harmonique correspondant $\ell p$ de la répartition $\mathbf{i}_{0}$ nous nous limiterons dans la suite au fondamental $(\ell=1)$ et omettrons cet indice dans les notations.

Nous prendrons ainsi $i_{0}$ sous la forme :

$$
\begin{aligned}
\mathbf{i}_{0}=\frac{1}{2} \mathbf{g}_{0}(r, z) \mathfrak{J}(t) & \exp -j p \theta+ \\
& +\frac{1}{2} \boldsymbol{g}_{0}^{*}(r, z) \mathfrak{J}^{*}(t) \exp j p \theta
\end{aligned}
$$

$\boldsymbol{g}_{0}$ est l'amplitude complexe du fondamental de la fonction de répartition des conducteurs du bobinage de la première phase de la génératrice [6] supposée triphasée. Nous avons posé :

$$
J(t)=\sum_{m=1}^{3} i_{m}(t) \exp j(m-1) \frac{2 \pi}{3}
$$

et l'astérisque désigne un nombre complexe conjugué.

$c_{n}^{\prime}(t)$ et $c_{n}^{\prime \prime}(t)$ étant dans (9) les coefficients relatifs à $\mathbf{j}_{n}^{\prime}$ et $\mathbf{j}_{n}^{\prime \prime}$, si nous groupons ces deux modes la série s'écrit :

$$
\mathbf{j}=\sum_{n=1}^{\infty} \mathbf{J}_{n}(r, z) C_{n}(t) \cos \left(\varphi_{n}(t)-p \theta\right)
$$

où

$$
\mathcal{C}_{n}(t)=c_{n}^{\prime}+j c_{n}^{\prime \prime}=C_{n}(t) \exp j \varphi_{n}(t) .
$$

La répartition de densité de courant relative au terme général de la nouvelle série (17) sera nommée dans la suite onde propre harmonique de polarité $p$ induite dans $\mathrm{D}$. Sa polarité $p$ étant connue elle est caractérisée par son amplitude complexe $\mathcal{C}_{n}(t) \mathbf{J}_{n}(r, z)$. Le coefficient $\mathcal{C}_{n}(t)$ est maintenant solution de l'équation différentielle : 


$$
\tau_{n} \frac{\mathrm{dC}_{n}}{\mathrm{~d} t}+\mathcal{C}_{n}=-\gamma \mathcal{A}_{0 n} \frac{\mathrm{d} \mathfrak{J}}{\mathrm{d} t}
$$

où

$$
\mathcal{A}_{0 n}=\pi \int_{d} \mathcal{A}_{0} \cdot \mathbf{J}_{n} r \mathrm{~d} r \mathrm{~d} z
$$

$\boldsymbol{A}_{0}$ est l'amplitude complexe [4] du potentiel vecteur donné par (3) et dû à la répartition (15) lorsque $J(t)$ est un courant constant égal à l'unité.

Dans le référentiel fixe lié au bobinage où nous allons maintenant nous placer, les équations (15) à (20) ont la même forme à l'exception de (19) dans laquelle il faut faire la substitution $\frac{\mathrm{d}}{\mathrm{d} t} \rightarrow\left(\frac{\mathrm{d}}{\mathrm{d} t}\right)$ - jp $\Omega$ et qui s'écrit alors

$$
\begin{aligned}
\tau_{n}\left(\frac{\mathrm{d}}{\mathrm{d} t}-j p \Omega\right) & \mathcal{C}_{n}+\mathcal{C}_{n}= \\
= & -\gamma \mathcal{A}_{0 n}\left(\frac{\mathrm{d}}{\mathrm{d} t}-j p \Omega\right) \mathfrak{J}(t) .
\end{aligned}
$$

Cela résulte du fait que les expressions des densités de courant dans le référentiel fixe s'obtiennent à partir de (15) et (17) en y effectuant la substitution $\theta \rightarrow \theta-\theta_{0}(t)$ où $\theta_{0}(t)$ est la position angulaire du rotor telle que $\Omega=\frac{\mathrm{d} \theta_{0}}{\mathrm{~d} t}$.

Le découplage constaté dans l'expression pour les pertes joule conduit à écrire :

$$
P_{\mathrm{J}}(t)=\sum_{n=1}^{\infty} \frac{1}{\gamma} \mathrm{C}_{n} \cdot \mathrm{C}_{n}^{*}
$$

Mais ici au niveau des ondes propres ce découplage se produit également pour la puissance mécanique fournie. En effet en portant la relation :

$$
\mathbf{v} \Lambda \operatorname{rot} \mathbf{a}_{0}=-\Omega \frac{\partial \mathbf{a}_{0}}{\partial \theta}+\Omega \operatorname{grad} r a_{0 \theta}
$$

dans l'expression suivante de la puissance mécanique fournie :

$$
P_{\mathrm{m}(\iota)}=\int_{\mathrm{D}}\left(\mathbf{j} \Lambda \operatorname{rot} \mathbf{a}_{0}\right) \cdot \mathbf{v} \mathrm{d} \tau
$$

on a :

$$
\begin{aligned}
P_{\mathrm{m}}(t) & =\sum_{n=1}^{\infty} \frac{1}{2} j p \Omega \times \\
& \times\left[\mathcal{C}_{n}(t) \mathcal{A}_{0 n}^{*} J^{*}(t)-\mathcal{C}_{n}^{*}(t) \mathcal{A}_{0 n} J(t)\right] .
\end{aligned}
$$

Notons que pour une excitation tournante telle que

$$
J(t)=\frac{3}{2} I \exp j \omega t
$$

où $I$ est l'amplitude commune des courants à variation sinusoïdale dans le temps de chacune des trois phases, les ondes propres harmoniques induites sont bien en régime permanent des ondes tournantes. En effet d'après (21) nous avons pour ces régimes permanents :

$$
\mathcal{C}_{n}(t)=-\frac{3}{2} I \frac{j \gamma g \omega}{1+j g \omega \tau_{n}} \mathcal{A}_{0 n} \exp j \omega t
$$

où nous avons posé $g \omega=\omega-p \Omega$. Ceci conduit bien à une onde tournante induite de même vitesse angulaire que l'excitation. Le calcul du rapport des expressions (22) et (25) conduit bien également à la relation puissance fréquence classique de la machine asynchrone en régime permanent soit :

$$
\frac{P_{\mathrm{m}}}{p \Omega}=\frac{P_{\mathrm{J}}}{g \omega}=\frac{P_{\mathrm{m}}+P_{\mathrm{J}}}{\omega} .
$$

Dans le cas du lanceur idéal de la figure 3 toutes les grandeurs vectorielles introduites dans (13) à (20) deviennent des scalaires dépendant uniquement de $r$ et portés par le vecteur unitaire $\frac{\mathrm{d} \mathbf{r}}{\mathrm{d} \boldsymbol{\theta}}$. On doit également substituer $k z$ à $p \theta$ et $k V$ à $p \Omega$. D'une façon plus précise (14) et (20) s'écrivent :

$$
\begin{gathered}
\int_{0}^{R} J_{n}^{2}(r) r \mathrm{~d} r=\frac{k}{2 \pi^{2}} \\
\mathcal{A}_{0 n}=\frac{2 \pi^{2}}{k} \int_{0}^{R} \mathcal{A}_{0}(r) J_{n}(r) r \mathrm{~d} r .
\end{gathered}
$$

5. Circuit équivalent associé à une onde propre $[6$, $18,9]$.

Nous nous placerons dans le cas de la génératrice triphasée de la figure 1, la transposition au lanceur étant immédiate. $i_{m}(t)$ et $v_{m}(t)$ représentant respectivement les courants et les tensions des phases nous effectuerons sur ces tensions et courants la transformation de Fortescue suivante :

$$
\begin{aligned}
\mathscr{X}(t) & =\sum_{m=1}^{3} x_{m}(t) \exp j(m-1) \frac{2 \pi}{3} \\
X^{*}(t) & =\sum_{m=1}^{3} x_{m}(t) \exp -j(m-1) \frac{2 \pi}{3} \\
X_{\mathrm{h}}(t) & =\sum_{m=1}^{3} x_{m}(t)
\end{aligned}
$$

où le couple $(\mathfrak{X}, x)$ sera identifié dans la suite à $(\mathfrak{J}, i)$ et $(\mathcal{V}, v)$

La loi d'Ohm en un point d'un conducteur de conductivité $\gamma_{0}$ de la $m$-ième phase du bobinage s'écrit :

$$
\frac{1}{\gamma_{0}} \mathbf{i}_{m}=-\frac{\partial \mathbf{a}}{\partial t}-\frac{\partial \mathbf{a}_{0}}{\partial t}-\operatorname{grad} u
$$

$\mathbf{i}_{m}, \mathbf{a}, \mathbf{a}_{0}$ et $u$ sont respectivement la densité de courant, les potentiels vecteurs induit et inducteur ainsi que le potentiel scalaire d'origine électrostatique. Après multiplication des deux membres de 
cette équation par $\mathbf{i}_{m}$ et intégration dans le volume $D_{0}$ nous obtenons les relations suivantes :

$$
\begin{aligned}
\mathcal{U}(t)= & R_{0} J(t)+ \\
& +L_{0} \frac{\mathrm{d} J}{\mathrm{~d} t}+\sum_{n=1}^{\infty} \frac{3}{2} \mathcal{A}_{0 n}^{*} \cdot \frac{\mathrm{dC}_{n}}{\mathrm{~d} t} \\
\mathcal{U}^{*}(t)= & R_{0} J^{*}(t)+ \\
& +L_{0} \frac{\mathrm{d} J^{*}}{\mathrm{~d} t}+\sum_{n=1}^{\infty} \frac{3}{2} \mathcal{A}_{0 n} \cdot \frac{\mathrm{dC}_{n}^{*}}{\mathrm{~d} t} \\
\mathcal{U}_{\mathrm{h}}(t)= & R_{0} J_{\mathrm{h}}(t)
\end{aligned}
$$

où

$$
\begin{aligned}
& R_{0}=\frac{3 \pi}{\gamma_{0}} \int_{d_{0}} g_{0} \cdot g_{0}^{*} r \mathrm{~d} r \mathrm{~d} z \\
& L_{0}=\frac{3 \pi}{2} \int_{d_{0}} A_{0} \cdot g_{0}^{*} r \mathrm{~d} r \mathrm{~d} z
\end{aligned}
$$

$d_{0}$ est la section méridienne de $\mathrm{D}_{0}$ et $\mathcal{C}_{n}(t)$ est donné à partir de $J(t)$ et de conditions initiales par l'équation différentielle (21).

Dans le cas où $\boldsymbol{\Omega}$ est constant, à partir des relations précédentes on met l'impédance cyclique opérationnelle de la machine asynchrone sans fer à rotor massif sous la forme suivante :

$$
\begin{aligned}
& Z(s)=\frac{V(s)}{I(s)}=R_{0}+ \\
& +s\left(L_{0}-(s-j p \Omega) \sum_{n=1}^{\infty} \frac{\frac{3}{2} \gamma \mathcal{A}_{0 n} \mathcal{A}_{0 n}^{*}}{1+\tau_{n}(s-j p \Omega)}\right)
\end{aligned}
$$

où $V(s)$ et $I(s)$ sont les transformées de Laplace respectives de $\mathcal{U}(t)$ et $J(t)$. Si on pose

$$
\frac{M_{n}}{L_{n}}=1 \quad \frac{M_{n}^{2}}{R_{n}}=\frac{3}{2} \gamma \mathcal{A}_{0 n} \mathcal{A}_{0 n}^{*} \quad \frac{L_{n}}{R_{n}}=\tau_{n}
$$

on peut alors déterminer $M_{n}, L_{n}, R_{n}$ et faire la synthèse [13] d'un circuit triphasé vérifiant les équations du système (3) lorsque $J_{h}(t)$ est nul. Nous donnons ce circuit sur la figure 4, circuit tel qu'il satisfait également (33) lorsque la vitesse angulaire $\Omega$ dépend $\mathrm{du}$ temps.

Les éléments de ce circuit sont ainsi définis pour $n>0$

$$
\begin{aligned}
L_{n} & =M_{n}=\frac{3}{2} \frac{\gamma \mathcal{A}_{0 n} \mathcal{A}_{0 n}^{*}}{\tau_{n}} \\
\ell_{n m} & =L_{n}
\end{aligned}
$$

et pour $n=0$

$$
\begin{gathered}
\ell_{0 m}=L_{0}-\sum_{n=1}^{\infty} L_{n} \\
{\left[\begin{array}{c}
E_{n 1} \\
E_{n 2} \\
E_{n 3}
\end{array}\right]=\frac{2}{3} p \Omega\left[\begin{array}{ccc}
0 & \frac{\sqrt{3}}{2} & -\frac{\sqrt{3}}{2} \\
-\frac{\sqrt{3}}{2} & 0 & \frac{\sqrt{3}}{2} \\
\frac{\sqrt{3}}{2} & -\frac{\sqrt{3}}{2} & 0 \\
\times\left[\begin{array}{cc}
i_{1} \\
i_{2} \\
i_{3}
\end{array}\right]+L_{n}\left[\begin{array}{c}
j_{n 1} \\
j_{n 2} \\
j_{n 3}
\end{array}\right]
\end{array}\right] \times}
\end{gathered}
$$

où les courants $j_{n m}$ pour $m$ variant de 1 à 3 sont définis sur la figure 4. A partir des variables figurant sur ce circuit on détermine ainsi les pertes joule de l'induit et la puissance mécanique foùrnie :

$$
\begin{aligned}
& P_{\mathrm{J}}(t)=\sum_{n=1}^{\infty} \sum_{m=1}^{3} R_{n} j_{n m}^{2} \\
& P_{\mathrm{m}}(t)=\sum_{n=1}^{\infty} \sum_{m=1}^{3} E_{n m} j_{n m} .
\end{aligned}
$$
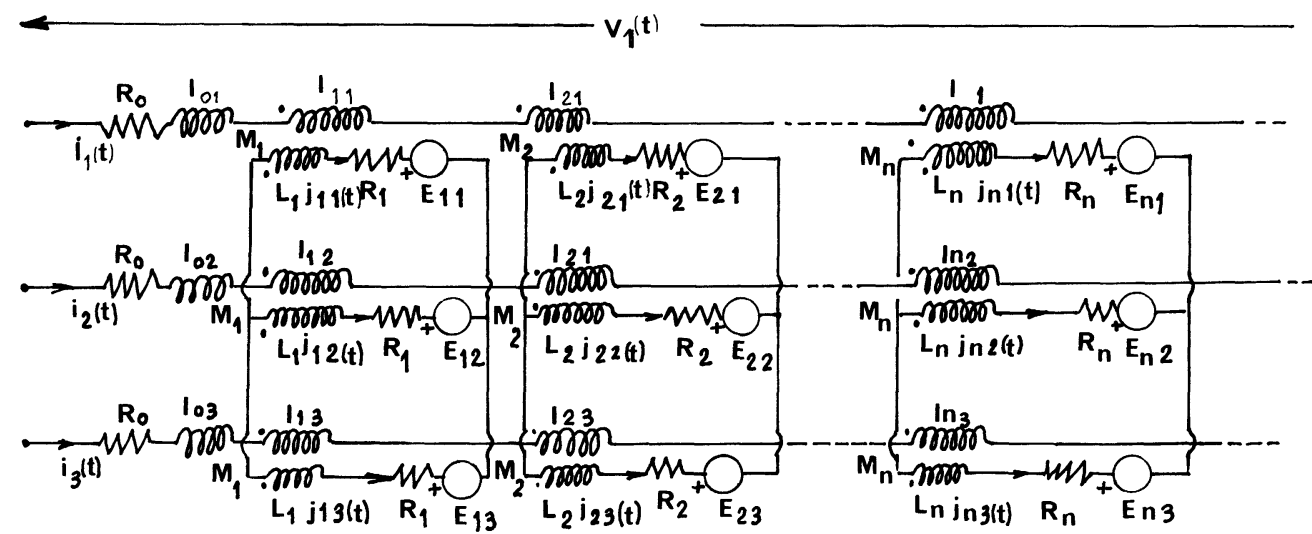

Mode propre $1 \quad$ Mode propre 2

\section{Constantes de temps $\tau_{1}$}

$\tau_{2}$

Mode propre $\mathbf{n}$

Fig. 4. - Circuit équivalent.

[Equivalent circuit.] 
Remarquons que dans ce schéma équivalent les circuits associés à chaque onde propre harmonique sont complètement découplés au point de vue électromécanique. On constate également qu'en régime harmonique permanent $(s=j \omega)$ la courbe de description de l'impédance cyclique relative à une onde harmonique est un cercle (Fig. 5). Le schéma équivalent de la machine asynchrone à rotor massif apparaît ainsi comme la mise en cascade de machines asynchrones idéales découplées. Les trois inductances de fuites $\ell_{0 m}$ constituant la dernière machine pour laquelle la constante de temps $\tau_{\infty}$ associée peut être considérée comme nulle.

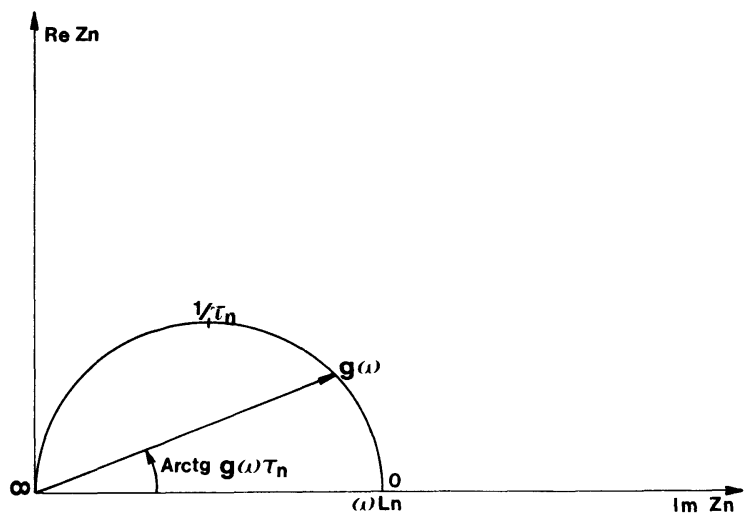

Fig. 5. - Courbe de description d'impédance de machine asynchrone idéale $Z_{n}(\omega, g \omega)=j \omega L_{n}\left(1-\frac{j g \omega \tau_{n}}{1+j g \omega \tau_{n}}\right)$.

[Impedance description curve of ideal asynchronous machine, $\left.Z_{n}(\omega, g \omega)=j \omega L_{n}\left(1-\frac{j g \omega \tau_{n}}{1+j g \omega \tau_{n}}\right).\right]$

Lorsque le courant homopolaire $\mathfrak{J}_{\mathrm{h}}(t)$ n'est pas nul la dernière relation (33) conduit à modifier le circuit précédent en prenant pour valeur de $\ell_{n m}$ les deux tiers de la valeur définie par (38) et (39) mais en couplant deux à deux ces inductances par une mutuelle inductance de valeur $-\frac{1}{3} \ell_{n m}$ où $\ell_{n m}$ est la nouvelle valeur adoptée.

\section{Applications aux génératrices impulsionnelles.}

Diverses techniques numériques peuvent être utilisées pour mettre en œuvre la méthode précédente. Ainsi dans le cas d'une maquette de génératrice impulsionnelle de type cylindrique nous avons utilisé [5] une méthode de calcul des puissances successives d'un opérateur $\overline{\overline{\mathcal{A}}}$ pour déterminer les constantes de temps $\tau_{n}$ et les coefficients $\mathcal{A}_{0} \cdot \mathcal{A}_{0 n}^{*}$ caractérisant les ondes propres excitées. Soit $\overline{\overline{\mathfrak{A}}}$ et $\overline{\overline{\mathfrak{A}}_{0}}$ les opérateurs associés respectivement à $\overline{\bar{a}}$ et $\overline{\bar{a}}_{0}$ mais agissant sur les amplitudes complexes [4]. Si nous posons :

$$
\mathbf{Y}_{0}=\overline{\overline{\mathcal{A}}}_{0}\left(\mathbf{g}_{0}\right)=\sum_{n=1}^{\infty} \mathcal{A}_{0 n} \mathbf{J}_{n}(r, z)
$$

et définissons le produit scalaire fonctionnel :

$$
\left\langle\mathfrak{F}_{1}, \mathfrak{F}_{2}\right\rangle=\pi \int_{d} \mathfrak{F}_{1}(r, z) \mathfrak{F}_{2}^{*}(r, z) r \mathrm{~d} r \mathrm{~d} z
$$

alors la suite de terme général $S_{\ell}$ :

$$
S_{\ell}=\left\langle\overline{\bar{A}}^{\ell}\left(\mathbf{y}_{0}\right), \mathbf{y}_{0}\right\rangle=\sum_{n=1}^{\infty} \mathcal{A}_{0 n} \mathcal{A}_{0 n}^{*} \lambda_{n}^{\ell}
$$

est une somme de suites géométriques de raisons décroissantes $\lambda_{n}$

La méthode que nous avons utilisée consiste à extraire successivement de $S_{\ell}$ ces suites. Ainsi $\ell$ étant suffisamment grand nous avons pris pour la première :

$$
\begin{gathered}
\lambda_{1}=\frac{S_{\ell+1}}{S_{\ell}} \quad \mathcal{A}_{01} \cdot \mathcal{A}_{01}^{*}=\frac{S_{\ell}}{\lambda_{1}^{\ell}} \\
\mathbf{J}_{1}(r, z)=\frac{\overline{\bar{A}}^{\ell}\left(\mathbf{y}_{0}\right)}{\lambda_{1}^{\ell}\left(\mathcal{A}_{01} \mathcal{A}_{01}^{*}\right)^{1 / 2}} .
\end{gathered}
$$

Puis considérant la nouvelle suite $S_{\ell}^{\prime}=S_{\ell}-$ $\mathcal{A}_{01} \mathcal{A}_{01}^{*} \lambda_{1}^{\ell}$ nous déterminons de la même façon $\lambda_{2}<\lambda_{1}$ et $\mathcal{A}_{02} \cdot \mathcal{A}_{02}^{*}$ caractérisant la seconde onde harmonique excitée et ainsi de suite. La perte de précision due aux soustractions est acceptable du fait de l'influence décroissante des ondes ainsi trouvées.

La mise en œuvre dans le cas (Fig. 6) d'un rotor cylindrique en duralumin $\left(\gamma=2 \times 10^{7}(\Omega . \mathrm{m})^{-1}\right)$ de

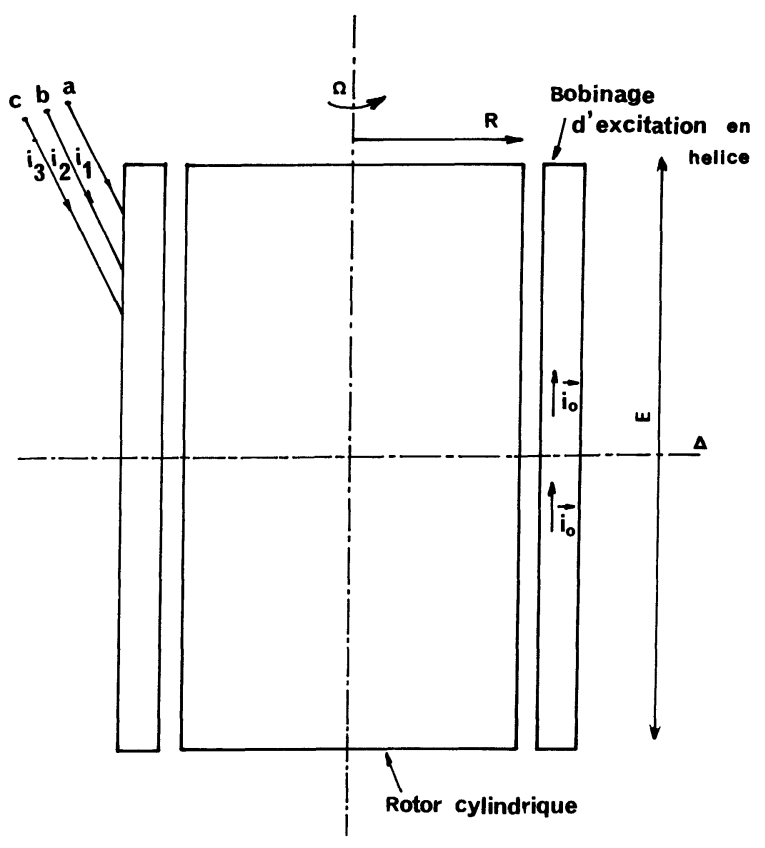

Fig. 6. - Génératrice impulsionnelle de type cylindrique.

[Cylindrical type pulsed generator.] 
rayon $R=0,148 \mathrm{~m}$ et de longueur $E=0,38 \mathrm{~m}$ placé à l'intérieur d'un bobinage triphasé à conducteurs en hélice et à 2 pôles nous a conduit $[5,6]$ à caractériser ainsi les deux premières ondes propres antisymétriques excitées :

$$
\begin{aligned}
\tau_{1} & =7,92 \times 10^{-2} \mathrm{~s} \\
\frac{3}{2} \gamma \mathcal{A}_{01} \mathcal{A}_{01}^{*} & =3,69 \times 10^{-6} \mathrm{H} . \mathrm{s} \\
\tau_{2} & =1,84 \times 10^{-2} \mathrm{~s} \\
\frac{3}{2} \gamma \mathcal{A}_{02} \mathfrak{A}_{02}^{*} & =6,36 \times 10^{-7} \text { H.s } .
\end{aligned}
$$

La figure 7 donne le schéma équivalent déduit de ces résultats numériques.

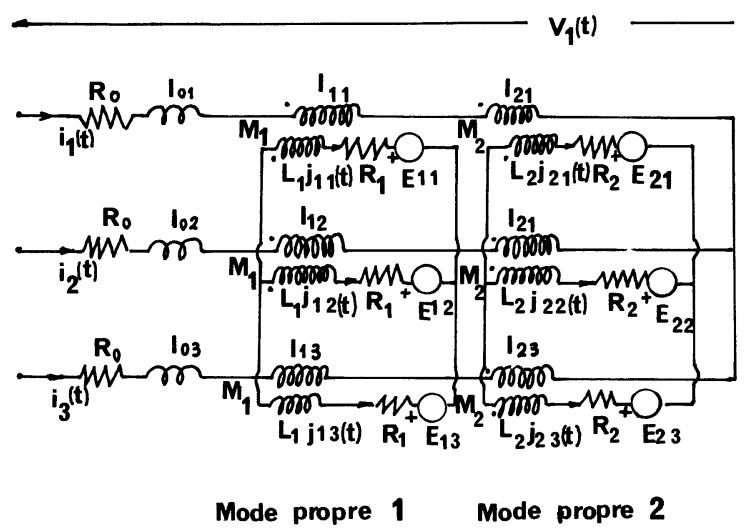

Fig. 7. - Circuit équivalent d'une génératrice de type cylindrique, $\quad R_{0}=0,035 \Omega, \quad \ell_{01}=38,9 \mu \mathrm{H}, \quad \ell_{11}=L_{1}=$ $M_{1}=46,6 \mu \mathrm{H}, \quad R_{1}=5,88 \times 10^{-4} \Omega, \quad \ell_{22}=L_{2}=$ $M_{2}=34,5 \mu \mathrm{H}, R_{2}=1,87 \times 10^{-3} \Omega$.

[Equivalent circuit of cylindrical generator type, $R_{0}=0.035 \Omega, \ell_{01}=38.9 \mu \mathrm{H}, \ell_{11}=L_{1}=M_{1}=46.6 \mu \mathrm{H}$, $R_{1}=5.88 \times 10^{-4} \Omega, \quad \ell_{22}=L_{2}=M_{2}=34.5 \mu \mathrm{H} ; \quad R_{2}=$ $1.87 \times 10^{-3} \Omega$.]

Une deuxième technique numérique consiste à utiliser une méthode de Galerkin dans laquelle on choisit a priori une base approchée de dimension finie pour $\mathcal{H}$. Dans ces conditions [11] la discrétisation associée de l'opérateur $\overline{\bar{A}}$ conduit à la recherche des valeurs propres et vecteurs propres d'une matrice. Nous avons ainsi mis en œuvre cette méthode [6] dans le cas de la génératrice impulsionnelle du Laboratoire (Fig. 8) à rotor discoïde en duralumin $\left(R=0,5 \mathrm{~m}, E=0,5 \mathrm{~m}, \quad \gamma=2 \times 10^{7}\right.$ $(\Omega . \mathrm{m})^{-1}$ ) et à bobinages plats en développante de cercle $\left(p=4,2 h=0,0126 \mathrm{~m}, \quad R_{i}=0,27 \mathrm{~m}\right)$. L'approximation des courants plans $\left(j_{z}=0\right)$ que nous avons utilisée a été justifiée au moyen du programme tridimensionnel de modélisation de courants de Foucault Trifou [10].

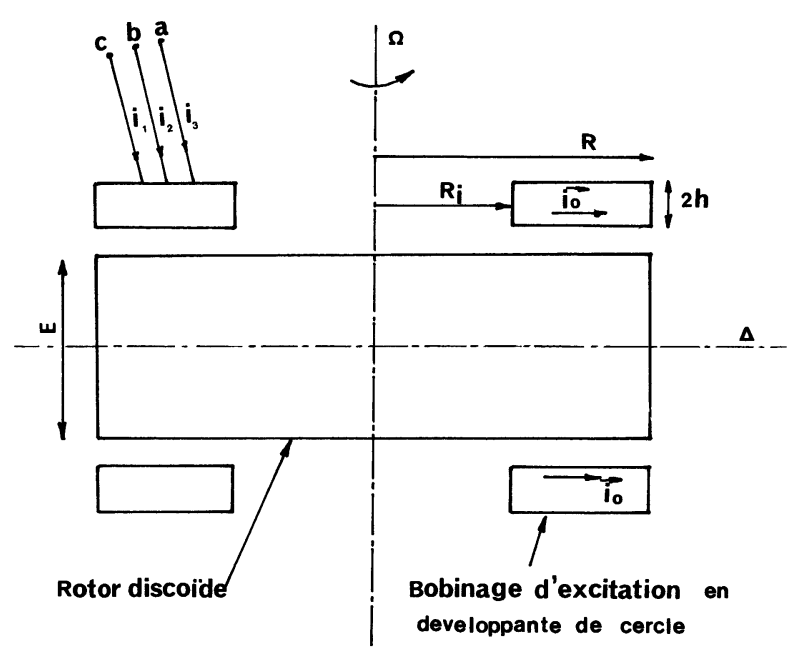

Fig. 8. - Génératrice impulsionnelle de type discoïde.

[Discoid type pulsed generator.]

Dans ces conditions [6] l'amplitude complexe $\boldsymbol{g}$ des courants induits n'a que deux composantes $j_{r}$ et $j_{\theta}$ qui sont définies par la connaissance de $\tilde{y}_{-}=g_{r}-j \dot{y}_{\theta}$. L'opérateur $\overline{\bar{A}}_{-}$associé à cette nouvelle variable $\boldsymbol{f}_{-}$et donnant la composante $(\overline{\overline{\mathfrak{A}}}(\boldsymbol{\gamma}))_{-}$ est alors l'opérateur intégral suivant [6] :

$$
(\overline{\overline{\mathfrak{A}}}(\boldsymbol{g}))_{-}=\frac{\mu_{0}}{4 \pi} \int_{d} \frac{F_{p+1}(\lambda) \cdot \mathcal{J}_{-}(u, v)}{\sqrt{(v-z)^{2}+u^{2}+r^{2}}} u \mathrm{~d} u \mathrm{~d} v
$$

où

$$
\begin{gathered}
F_{p+1}(\lambda)=\int_{0}^{2 \pi} \frac{\cos (p+1) \theta}{\sqrt{1-\lambda \cos \theta}} \mathrm{d} \theta \\
\lambda=\frac{2 u r}{(v-z)^{2}+u^{2}+r^{2}} .
\end{gathered}
$$

Nous avons pris comme base des $g_{-}(r, z)$ dans la section méridienne $d$ les fonctions suivantes [6] déduites des modes propres symétriques indépendants en $z$ du rotor infiniment épais soit :

$$
I_{n^{\prime}, n^{\prime \prime}}=J_{p+1}\left(r \beta_{n^{\prime}}\right) P_{2 n^{\prime \prime}}(z)
$$

$J_{p+1}(x)$ est la fonction de Bessel d'ordre $p+1$ et $R \beta_{n^{\prime}}$ le $n^{\prime}$-ième zéro de $J_{p}(x)$ et $P_{2 n^{\prime \prime}}$ le polynôme de Legendre sur $\left[-\frac{E}{2}, \frac{E}{2}\right]^{p}$ de degré $2 n^{\prime \prime}$. En prenant $n^{\prime} \leqslant 3$ et $n^{\prime \prime} \leqslant 2$ nous avons ainsi déterminé six ondes propres dont les caractéristiques sont données dans le tableau I. Sur les figures 9 à 11 nous donnons, à une constante multiplicative près et dans le plan médian du rotor $(z=0)$, les champs de flèches représentant les densités de courant des trois premiers modes propres excités. Toujours pour $z=0$ nous donnons sur la figure 12 les densités de courant induit en régime sinusoïdal permanent $(g \omega=$ 
Tableau I. - Caractéristiques de six ondes propres d'une génératrice impulsionnelle.

[Six proper wave characteristics of pulsed generator.]

\begin{tabular}{|c|c|c|}
\hline$n$ & $\tau_{n}$ en s & $\frac{3}{2} \gamma \mathcal{A}_{0 n} \mathcal{A}_{0 n}^{*}$ en H.s \\
\hline 1 & $3,25 \times 10^{-2}$ & $1,05 \times 10^{-5}$ \\
\hline 2 & $2,07 \times 10^{-2}$ & $2,61 \times 10^{-6}$ \\
\hline 3 & $1,49 \times 10^{-2}$ & $1,63 \times 10^{-7}$ \\
\hline 4 & $2,90 \times 10^{-3}$ & $3,79 \times 10^{-8}$ \\
\hline 5 & $2,79 \times 10^{-3}$ & $4,97 \times 10^{-8}$ \\
\hline 6 & $2,64 \times 10^{-3}$ & $3,84 \times 10^{-8}$ \\
\hline
\end{tabular}

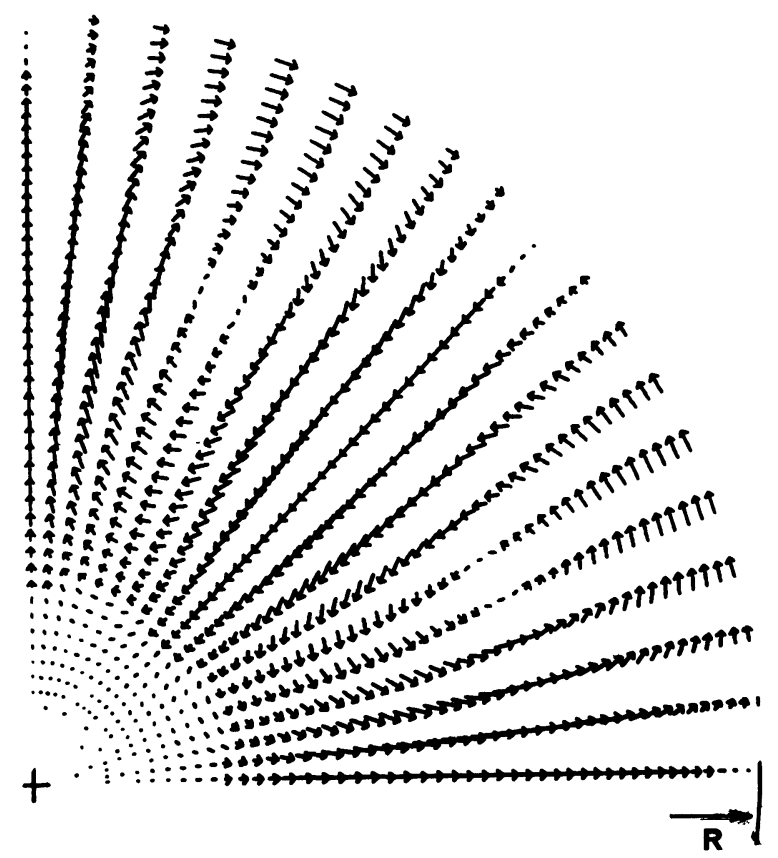

Fig. 9. - Densité de courant du premier mode $(n=1)$. [Current density of mode one.]

$20 \mathrm{rd} / \mathrm{s})$ obtenues par sommation des six ondes propres. Les densités de courant d'excitation à la pulsation $\omega$ ayant au même instant une répartition moyenne dans $\mathrm{D}_{0}$ donnée sur la figure 13 . La courbe de description de $G(g \omega)+j H(g \omega)$ définissant l'impédance cyclique de la génératrice déduite de (35) :

$$
Z(\omega, g \omega)=R_{0}+\omega(G(g \omega)+j H(g \omega))
$$

est portée sur la figure 14 ainsi que la contribution des trois premières ondes propres.

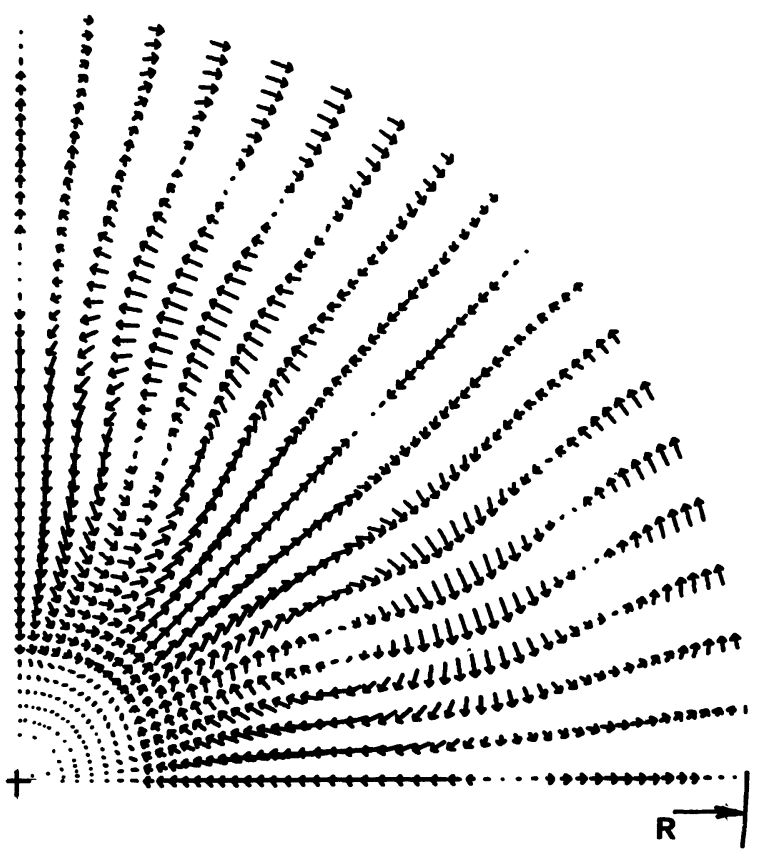

Fig. 10. - Densité de courant du deuxième mode $(n=2)$.

[Current density of mode two.]

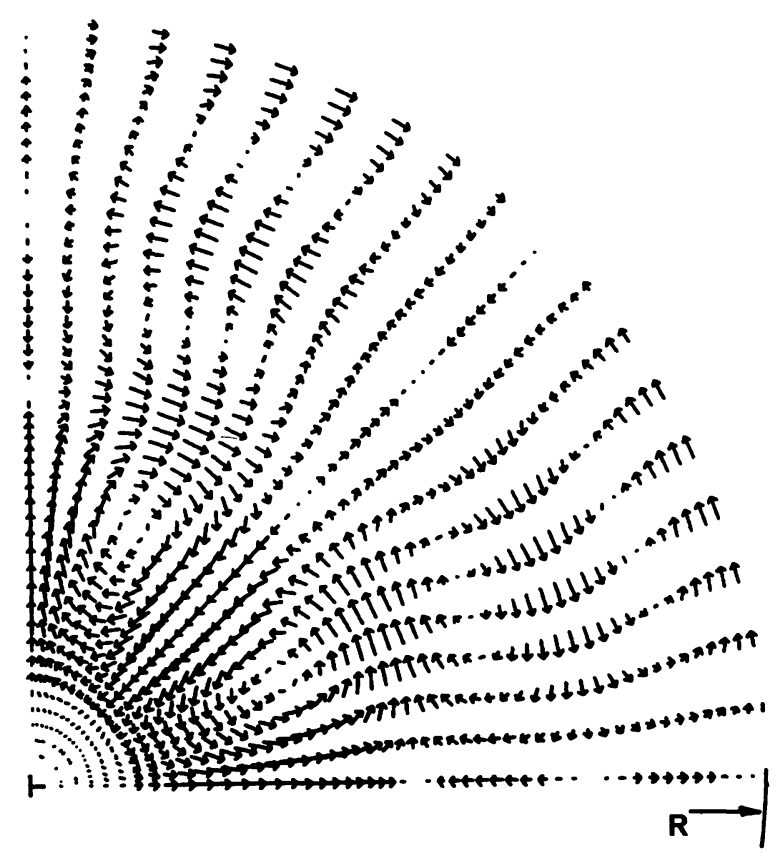

Fig. 11. - Densité de courant du troisième mode $(n=3)$.

[Current density of mode three.]

\section{Application aux lanceurs électromagnétiques [12,} 19].

Considérons enfin le cas idéal du lanceur à inducteur triphasé de la figure 3 pour lequel la densité des spires d'une phase du bobinage d'excitation est à 


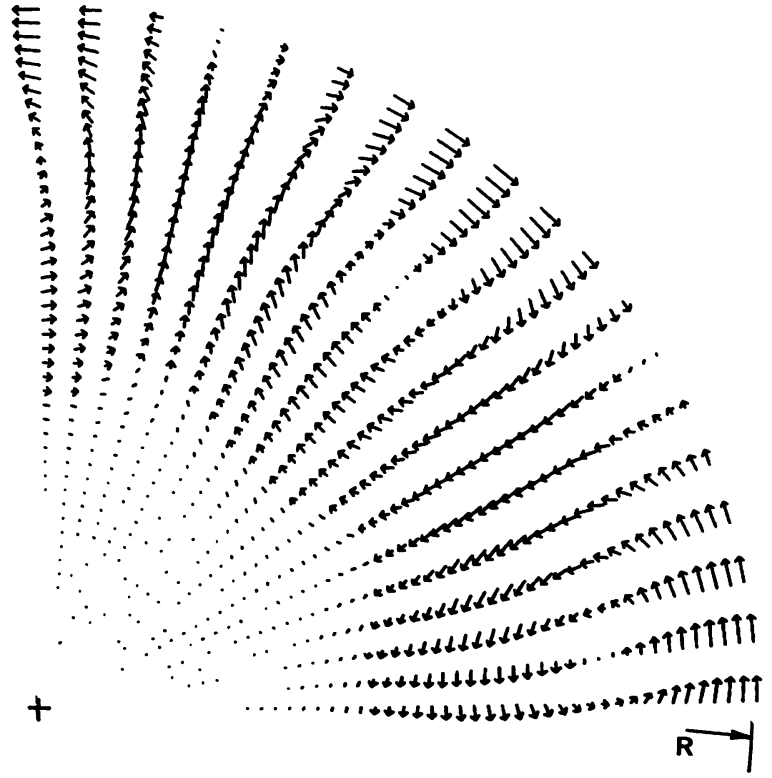

Fig. 12. - Densité des courants induits $(g \omega=20 \pi \mathrm{rd} / \mathrm{s}$, $z=0$, densité maximale $\left.=0,42 \times 10^{6} \mathrm{~A} / \mathrm{m}^{2}\right)$.

[Induced currents density $(g \omega=20 \pi \mathrm{rd} / \mathrm{s}, z=0$, maximal density $\left.=0.42 \times 10^{6} \mathrm{~A} / \mathrm{m}^{2}\right)$.]

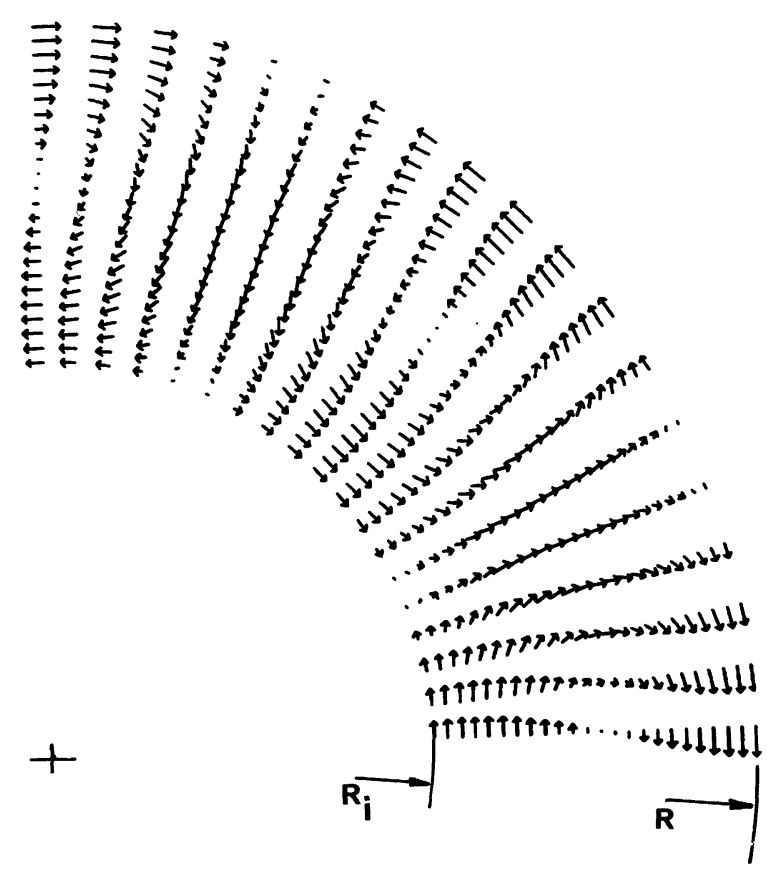

Fig. 13. - Densité de courant moyenne dans le bobinage d'excitation (densité maximale $=0,16 \times 10^{7} \mathrm{~A} / \mathrm{m}^{2}$ ).

[Mean current density in excitation winding (maximal density $\left.=0.16 \times 10^{7} \mathrm{~A} / \mathrm{m}^{2}\right)$.]

répartition sinusoïdale en $z$ de période $\frac{2 \pi}{k}$. Dans ces conditions l'amplitude complexe de la densité de courant d'excitation du volume cylindrique $D_{0}$ compris entre les rayons $R_{1}$ et $R_{2}$ est portée par

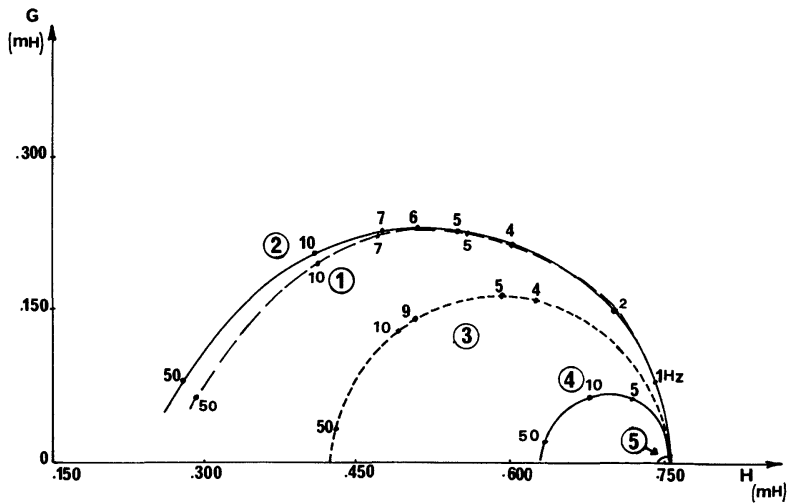

Fig. 14. - Courbe de description $G(g \omega)+j H(g \omega)$, 1]d'après référence [3], 2]sommation des six ondes, 3 premier mode, 4 deuxième mode, 5 troisième mode.

[Description curve $G(g \omega)+j H(g \omega)$, 1 from reference [3], 2]summation of six waves, 3 first mode, 4]second mode, [5third mode.]

$\frac{\mathrm{d} \mathbf{r}}{\mathrm{d} \theta}$ et a pour expression :

$$
\mathfrak{J}_{0}(r, t)=\mathfrak{J}(t) \mathfrak{S}_{0}(r)
$$

où $\mathfrak{J}(t)$ est toujours donné par (26). La recherche des ondes propres excitées s'effectue au moyen des amplitudes complexes

$$
\mathfrak{A}_{n}(r, t)=\exp -\frac{t}{\tau_{n}} \mathfrak{A}_{n}(r)
$$

de potentiels vecteurs vérifiant le problème aux limites suivant où la conductivité $\gamma$ est nulle pour $r>R$ :

$$
\mid \begin{aligned}
& \frac{\mathrm{d}^{2} \mathcal{A}_{n}}{\mathrm{~d} r^{2}}+\frac{1}{r} \frac{\mathrm{d} \mathcal{A}_{n}}{\mathrm{~d} r}-\left(\frac{1}{r^{2}}+k^{2}\right) \mathcal{A}_{n}=-\frac{\mu_{0} \gamma}{\tau_{n}} \mathcal{A}_{n} \\
& \text { continuité de } \mathcal{A}_{n} \text { et } \frac{\mathrm{d} \mathfrak{A}_{n}}{\mathrm{~d} r} \text { en } r=R .
\end{aligned}
$$

Les ondes propres excitées sont alors ainsi caractérisées :

$$
\begin{gathered}
\tau_{n}=\frac{\mu_{0} \gamma R^{2}}{R^{2} \beta_{n}^{2}+k^{2} R^{2}} \\
J_{n}(r)=\frac{\sqrt{k}}{\pi R} \frac{\beta_{n} R}{\sqrt{\beta_{n}^{2} R^{2}-1+h^{2}}} \frac{J_{1}\left(\beta_{n} r\right)}{J_{1}\left(\beta_{n} R\right)}
\end{gathered}
$$

où $\beta_{n} R$ est racine positive de l'équation :

$$
\begin{aligned}
k R K_{0}(k R) J_{1}\left(\beta_{n} R\right)+ & \\
& +\beta_{n} R J_{0}\left(\beta_{n} R\right) K_{1}(k R)=0
\end{aligned}
$$

et

$$
h=1+k R \frac{K_{0}(k R)}{K_{1}(k R)}
$$

$J_{0}, J_{1}$ sont les fonctions de Bessel et $I_{0}, K_{0}$, $I_{1}, K_{1}$ les fonctions de Bessel modifiées [14]. 
L'amplitude complexe du potentiel vecteur d'excitation étant, dans le volume de l'induit, proportionnelle à $I_{1}(k r)$ on a après calcul de l'intégrale (30) :

$$
\begin{aligned}
\mathcal{A}_{0 n}=\mathcal{B}_{0} \frac{\sqrt{k} k R K_{0}(k R)}{\pi \mu_{0} \gamma R}( & \left.\frac{I_{0}(k R)}{K_{0}(k R)}+\frac{I_{1}(k R)}{K_{1}(k R)}\right) \times \\
& \times \frac{1}{\sqrt{1+\frac{h^{2}-1}{\beta_{n}^{2} R^{2}}}} \tau_{n}
\end{aligned}
$$

où

$$
\mathfrak{B}_{0}=\mu_{0} \int_{R_{1}}^{R_{2}} K_{1}(k r) \mathfrak{G}_{0}(r) r \mathrm{~d} r .
$$

L'application numérique pour laquelle nous avons pris $k R=1$ conduit aux résultats portés sur le tableau II. Ainsi comme dans le cas de la génératrice impulsionnelle les deux ondes propres excitées possédant les plus grandes constantes de temps sont suffisantes pour étudier un tel lanceur.

Tableau II. - Caractéristiques de quatre ondes propres d'un lanceur idéal.

[Four proper wave characteristics of ideal launcher.]

\begin{tabular}{|c|l|c|c|c|}
\hline$n$ & 1 & 2 & 3 & 4 \\
\hline$\beta_{n} R$ & 2,76 & 5,71 & 8,80 & 11,9 \\
\hline$\tau_{1} / \tau_{n}$ & 1 & 3,90 & 9,1 & 16,5 \\
\hline$\frac{\mathfrak{t}_{0 n} \cdot \mathfrak{A}_{0 n}^{*}}{\mathfrak{A}_{01} \cdot \mathcal{A}_{01}^{*}}$ & 1 & $7,91 \times 10^{-2}$ & $1,51 \times 10^{-2}$ & $4,67 \times 10^{-3}$ \\
\hline
\end{tabular}

L'onde de plus grande constante de temps $\tau_{1}$ étant déjà représentative du fonctionnement du lanceur nous avons pour cette onde déterminé le rapport de l'énergie cinétique transférée à l'induit sur l'énergie perdue par effet joule dans cet induit. Pour cela nous avons supposé que l'établissement des courants inducteurs est instantané et conduit dans le référentiel mobile à :

$$
\begin{array}{ll}
J(t)=0 & t<0 \\
J(t)=\frac{3}{2} I \exp j g \omega t & t>0 .
\end{array}
$$

Grâce à une régulation on suppose que la pulsation $g \omega$ de ces courants induits est constante pendant la propulsion à la vitesse variable $V(t)$. Dans ces conditions (19) conduit pour $t>0$ à :

$$
\begin{aligned}
\mathcal{C}_{1}(t)=- & \frac{3 \gamma \mathcal{A}_{01} I}{2 \tau_{1}\left(1+j g \omega \tau_{1}\right)} \times \\
& \times\left(j g \omega \tau_{1} \exp j g \omega t+\exp -\frac{t}{\tau_{1}}\right) .
\end{aligned}
$$

On peut alors calculer au moyen de (22) et (25) les expressions

$$
\begin{aligned}
& \frac{F(t)}{F(\infty)}=1-\left(\cos g \omega t+\frac{1}{g \omega \tau_{1}} \sin g \omega t\right) \exp -\frac{t}{\tau_{1}} \\
& \frac{P_{\mathrm{J}}(t)}{P_{\mathrm{J}}(\infty)}=1-\frac{2}{g \omega \tau_{1}} \sin g \omega t \exp -\frac{t}{\tau_{1}}+ \\
& +\frac{1}{\left(g \omega \tau_{1}\right)^{2}} \exp -\frac{2 t}{\tau_{1}}
\end{aligned}
$$

de l'effort $F(t)$ et de la puissance perdue par effet joule relatifs à l'induit comparées à leurs expressions $F(\infty)$ et $P_{\mathrm{J}}(\infty)$ en régime sinusoïdal permanent. Nous avons alors défini un coefficient d'efficacité asynchrone $\eta\left(g \omega \tau_{1}, g \omega t\right)$ tel que :

$$
\eta=\frac{2 g \omega}{k V(t)} \frac{W_{\mathrm{c}}}{W_{\mathrm{J}}}=\frac{\int_{0}^{t} \frac{F(u)}{F(\infty)} \mathrm{d} u}{\int_{0}^{t} \frac{P_{\mathrm{J}}(u)}{P_{\mathrm{J}}(\infty)} \mathrm{d} u}
$$

$W_{c}$ est l'énergie cinétique $\frac{1}{2} M V^{2}(t)$ transférée à l'induit, $W_{\mathrm{J}}$ l'énergie perdue par effet joule et $\frac{k V(t)}{2 g \omega}$ la valeur en régime permanent du rapport $\frac{2 g \omega}{W_{c}}$

$\frac{W_{\mathrm{c}}}{W_{\mathrm{J}}}$ obtenu à partir de (28). Sur la figure 15 nous avons porté pour cinq valeurs de $g \omega \tau_{1}$ les variations du coefficient d'efficacité asynchrone en fonction de $\frac{t}{T}$ où $T=\frac{2 \pi}{g \omega}$ est la période des courants induits. Une étude en régime permanent [19] conduisant à prendre $g \omega \tau_{1}$ voisin de l'unité nous voyons sur cette

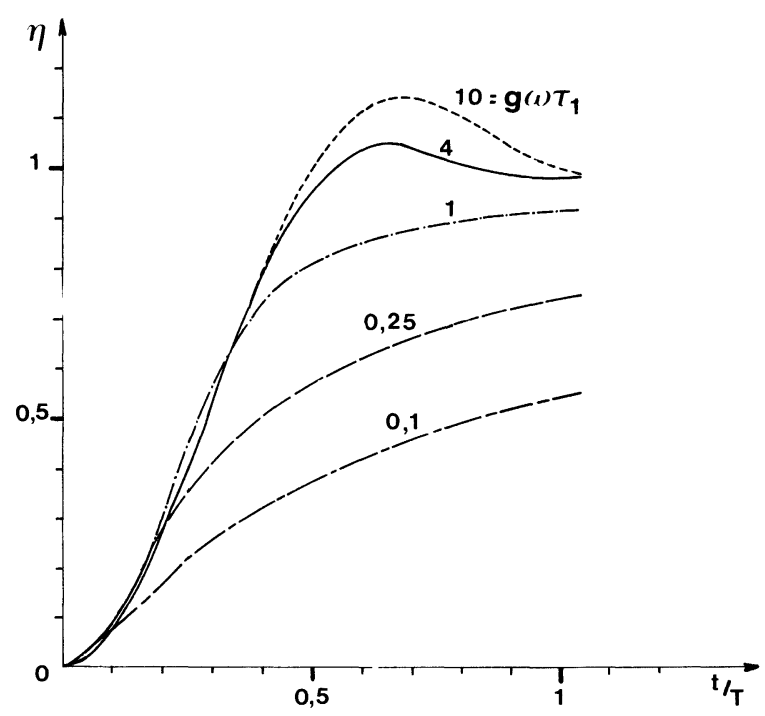

Fig. 15. - Coefficient d'efficacité asynchrone pour un lanceur électromagnétique idéal.

[Asynchronous efficiency coefficient of an ideal electromagnetic launcher.] 
figure que $\eta$ ne sera proche de sa valeur asymptotique unité que si la mise en vitesse s'effectue en un temps supérieur à la période $T$ des courants induits.

\section{Conclusion.}

La décomposition des courants induits en modes propres caractérisés chacun par une constante de temps et une densité de répartition est particulièrement intéressante lorsqu'on peut grouper ces modes en ondes propres harmoniques. En effet dans ce cas on est conduit à un découplage électromécanique complet de ces ondes. On peut également associer à chacune d'elles un circuit équivalent simple de machine asynchrone idéale dont le diagramme d'impédance est circulaire, le circuit équivalent complet étant obtenu par la mise en cascade de tels circuits élémentaires. Enfin nous avons également constaté dans le cas de génératrice impulsionnelle où de lanceur à induit long que la prise en compte de deux ou trois ondes excitées était suffisante pour étudier le dispositif.

\section{Bibliographie}

[1] Sultanem, F., Bleiss, C. A., Rioux, C., RiouxDAMIDAU, F., Study of a pulsed générator based on an iron-free asynchronous machine. J. Appl. Phys., 52 (1982) 3655-3662.

[2] Rioux, F., Calcul semi-tridimensionnel des champs et des courants dans les machines cylindriques asynchrones sans fer. Revue Phys. Appl. 18 (1983) 113-127.

[3] Ly, L., Quichaud, G., Sultanem, F., Simulation numérique globale de la décharge impulsionnelle d'une génératrice asynchrone sans fer. Revue Phys. Appl. 15 (1980) 1625-1630.

[4] Tchakala, E., Quichaud, G., Modeling of ironfree electrical machines with solid rotors using a magnetic vector potential formulation. Electr. Mach. Electromech. 7 (1982) 243-260.

[5] Andriamanana, E., Diop, A., Quichaud, G., Détermination des modes propres de diffusion des courants dans un rotor cylindrique amagnétique en rotation uniforme. Colloque MODELEC, La Grande-Motte, 22-24 octobre 1984, 112.

[6] Diop, A., Modélisation des machines asynchrones sans fer à rotor massif adaptée à l'étude de leurs régimes transitoires. Thèse de Docteur-Ingénieur, Université Paris VI, 1985.

[7] Sultanem, F., Les machines asynchrones sans fer à rotor massif. Caractéristiques générales et utilisations en génératrices impulsionnelles. Thèse de Docteur-ès-Sciences, Paris 1981.

[8] Bossavit, A., Le problème des courants de Foucault. Journée d'étude. Méthodes Numériques en Electronique et en Electrotechnique, INRIA, 1-11 (janvier 1980).

[9] Boyer, L., Fournet, G., Study of screens for superconducting a.c. machines. Electr. Mach. Electromech. 4 (1979) 359-370 et 5 (1980) 77-85.
[10] Vérité, J., Chaussecourte, P., Rose, C. ANDRIAMANANA, E., Gros, M. : TRIFOU, Un code de calcul tridimensionnel des courants de Foucault. Colloque MODELEC, La GrandeMotte, 22-24 octobre 1984, 343-358.

[11] Krasnov, Kisselev, MAKARENKo, Equations intégrales (Editions de Moscou).

[12] Rioux, C., Rioux-Damidau, F., Lanceurs électromagnétiques. Journées d'étude SEE, Centrales solaires spatiales, 5-6 juin 1986, p. 96-99.

[13] Quichaud, G., Analyse des circuits électriques par ordinateur, Cours DEA d'Electrotechnique, Paris 6.

[14] ANgot, A., Compléments de Mathématiques à l'usage des ingénieurs de l'électrotechnique et des télécommunications (Masson).

[15] Silvester, P., Konrad, A., Coulomb, J. L., SABONNAdiere, J. C., Modal network representation of slot-embedded conductors. Electr. Mach. Electromech. 1 (1977) 107-122.

[16] WeIss, J., CsEndes, Z. J., Finite element solution of eddy currents in asynchronous generator rotors. Proceedings of ICEM GP1/3, Athènes 1980, 1511-1518.

[17] Silvester, P., Eddy current modes in linear solidiron bars, Proceedings of I.E.E. 112 (1965) 15891594.

[18] $\mathrm{KoCH}$, W., Equivalent circuits with transformer elements for eddy current rotor induction motors derived from field equations. Part I : Sleeve rotor motors, Part II : Drag cup rotor motors, I.E.E.E. Trans. Power Appar. Syst. 83 (1964) 567-583.

[19] LuCHET, J. C., Modélisation d'un lanceur électromagnétique à induction en régime permanent et approche de l'optimisation. Rapport stage DEA d'Electrotechnique, juin 1987, Paris 6. 